\title{
Una aproximación a lo cualitativo: identificando las creencias de la compartición de conocimiento en las comunidades de práctica
}

An approach to qualitative feature: identifying the beliefs of knowledge sharing in practicing communities

Uma aproximação ao qualitativo: identificando crenças do compartilhamento de conhecimento nas comunidades de prática

\author{
Mariela Isabel Oviedo Santillán \\ Universidad Andina Simón Bolívar, Sede Ecuador \\ marielaisab@gmail.com \\ https://orcid.org/0000-0002-2093-3049 \\ Marcelo Fernando López Parra \\ Universidad Andina Simón Bolívar, Sede Ecuador \\ ferlopezparra@gmail.com \\ https://orcid.org/0000-0002-3948-5127
}

DOI: https://doi.org/10.32719/25506641.2020.7.1

Recibido: 15 de agosto de 2019 • Revisado: 16 y 20 de octubre de 2019

Aceptado: 14 de noviembre de 2019

Artículo de investigación

Licencia Creative Commons

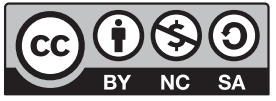




\section{Resumen}

Este trabajo documental y exploratorio hace énfasis en un abordaje cualitativo como una fase previa y complementaria de lo cuantitativo. El estudio se presenta en la aplicación de un caso práctico y el uso de la teoría del comportamiento planificado para identificar las creencias conductuales, normativas y de control que influyen en la motivación de los individuos para compartir su conocimiento en las comunidades de práctica de las organizaciones públicas. Para recolectar datos al respecto, se entrevistó a expertos de la administración tributaria en la ciudad de Quito, Ecuador. Los datos fueron clasificados y codificados en vocablos con miras al reconocimiento de 63 creencias que, en un futuro y luego de una priorización, permitirán la construcción de un cuestionario que valide las relaciones entre las variables del modelo de motivación empleado. En la revisión documental, se contraponen las fortalezas de lo cualitativo y cuantitativo, se desecha su incompatibilidad y se aboga por la selección de las aproximaciones híbridas, resaltando lo mejor de cada método y ejemplificando la utilización de los métodos cualitativos en la construcción de tipologías de narraciones de casos, así como de las narrativas modales como categorías en el análisis cuantitativo.

Palabras clave: Investigación cualitativa, métodos mixtos, comunidades de práctica, compartición de conocimiento, motivación, administración pública.

JEL: D23 Comportamiento organizativo; costes de transacción; derechos de propiedad.

\section{Abstract}

This documentary and exploratory work emphasizes a qualitative approach as a preliminary and complementary phase of the quantitative. The study is presented in the application of a case and the use of the Theory of Planned Behaviour to identify behavioural, normative and control beliefs that influence the motivation in individuals to share their knowledge in communities of public organizations. For this, data was collected from applied interviews to experts of the Tax Administration in Quito-Ecuador. The data were classified and codified in words, expecting the recognition of 63 beliefs which will allow the construction of a questionnaire in the future that validates the relationships between the variables of the motivation model used. In the documentary review, the strengths of qualitative and quantitative are contrasted, their incompatibility is rejected and the selection of hybrid approaches is encouraged, highlighting the best of each method and exemplifying the use of qualitative methods in the construction of typologies of case narratives and modal narratives as categories in the quantitative analysis.

Keywords: Qualitative research, mixed methods, communities of practice, knowledge sharing, motivation, public administration.

JEL: D23 Organizational behaviour; transaction costs; property rights.

\section{Resumo}

Este trabalho documental e exploratório enfatiza uma abordagem qualitativa como uma fase prévia e complementar do quantitativo. O estudo se compõe da aplicação de um caso 
prático e do uso da Teoria do Comportamento Planificado para identificar crenças condutuais, normativas e de controle que influem na motivação dos indivíduos para compartilharem seu conhecimento nas comunidades de prática das organizações públicas. Para tal, utilizou-se a entrevista para coletar dados, aplicada a especialistas da Administração Tributária na cidade de Quito-Equador. Os dados foram classificados e codificados em vocábulos, com vista ao reconhecimento de 63 crenças que, em um futuro e após uma priorização, permitirão a construção de um questionário que valide relações entre variáveis do modelo de motivação empregado. Na revisão documental, contrapõem-se pontos fortes do qualitativo e quantitativo, descarta-se sua incompatibilidade e se advoga pela seleção de aproximações híbridas, ressaltando o melhor de cada método e exemplificando a utilização dos métodos qualitativos na construção de tipologias de narrações de casos e narrativas modais como categorias na análise quantitativa.

Palavras-chave: Pesquisa qualitativa, métodos mistos, comunidades de prática, compartilhamento de conhecimento, motivação, administração pública.

JEL: D23 Comportamento organizativo; custos de transação; direitos de propriedade.

\section{Introducción}

L as discusiones sobre una división simplista de los métodos cualitativos contra los métodos cuantitativos ahora han sido reemplazadas por la atención en las implicaciones de diferentes lógicas para la investigación y el desarrollo del conocimiento (Haverland y Yanow 2012). Esto se refleja en el crecimiento exponencial de la investigación cuantitativa, acompañada del desarrollo paralelo y continuo de su homóloga (Cassell y Symon 2015).

En este contexto, lo cualitativo se puede ver como una metodología bien establecida capaz de responder a grandes preguntas de la investigación y útil para fortalecer los vínculos teórico-prácticos, lo que es consistente con la explosión de reflexiones sobre estudios y metodología cuantitativa en las ciencias sociales y administrativas en los últimos años.

La metodología cualitativa tiene una larga historia y tradición dentro de la investigación de la organización y la gestión. Aportando a la comprensión de la experiencia y la práctica gerencial, las primeras etnografías sobre el trabajo directivo datan de los años 50, 70 y 80, con trabajos como Men Who Manage, de Dalton (1959); The Personnel Managers, de Watson (1977); y Moral Mazes: The World of Corporate Managers, de Jackall (1988). Más 
adelante, se argumentó que las técnicas cualitativas pueden -en generalproporcionar herramientas poderosas en la investigación de la gestión y la organización Cassell y Symon 2006).

Actualmente se pueden encontrar en todos los dominios de la administración trabajos cualitativos que cubren los diversos campos de la organización, la gestión y el marketing (Cassell y Symon 2006).

A pesar de este surgimiento en el campo empírico, es innegable el predominio de las investigaciones cuantitativas (Ospina, Esteve y Lee 2017). Entonces, es obligatorio asegurar que ambos tipos de metodología tengan la misma posición con respecto a la legitimidad de las afirmaciones de conocimiento generadas por la investigación empírica (Ospina, Esteve y Lee 2017). Ejemplificar su uso - tal y como se pretende hacer aquí- y mejorar la calidad de los informes de investigación cualitativos pueden ayudar a este objetivo (Ashworth, McDermott y Currie 2018).

Ahora bien, las críticas de lo cualitativo ya no giran en torno a las ventajas y desventajas con respecto a su par -lo cuantitativo-, sino en torno a que los reportes e informes generados son opacos (Ospina, Esteve y Lee 2017) y a que existe la necesidad de realizar elecciones explícitas, consistentes y transparentes a lo largo de todo el proceso de investigación (Dodge, Ospina y Foldy 2005).

Esto hace que los reclamos de transparencia se relacionen con un mejor diseño de la investigación, la transición de la recopilación de datos al análisis, y cómo la información sustenta la contribución. Sin embargo, el acuerdo sobre los criterios de evaluación estándar resultan ser un desafío por la pluralidad de los enfoques cualitativos (Ospina, Esteve y Lee 2017).

A pesar de que los estudios cuantitativos también muestran la diversidad metodológica, las diferencias filosóficas subyacentes y evidentes de los métodos cualitativos dificultan la aplicación de una plantilla genérica para evaluar su rigor científico, especialmente cuando no es fácil aplicar pruebas estándar de validez y fiabilidad a los enfoques cualitativos (Dodge, Ospina y Foldy 2005). Por lo tanto, existe la preocupación de que los juicios de la investigación cualitativa a menudo se basan en una definición problemática de calidad y criterios equivocados; consecuentemente, los estudiosos aceptan una versión de rigor de manera que se reduzca la pluralidad de la investigación cualitativa, o se rechace el concepto para socavar su legitimidad. Esto sugiere 
que es necesario lograr un mejor equilibrio entre rigor y riqueza, y desarrollar principios más amplios, de modo que la investigación cualitativa sustente la teorización y se adapte a la pluralidad (Dodge, Ospina y Foldy 2005).

Por otro lado, el éxito del método utilizado -cualitativo o cuantitativodependerá de la naturaleza del trabajo académico. No tiene sentido preguntarse si una metodología es mejor que otra: habrá investigaciones que hagan uso de un solo método, pero en otras oportunidades se requerirá de ambas opciones, es decir, se seleccionarán y utilizarán técnicas de investigación empíricas y cuantitativas. No existe la necesidad de optar por una de las dos aproximaciones, sino más bien determinar cómo se pueden combinar las fortalezas de cada enfoque, lo que permitirá enriquecer y ahondar en los resultados de las propuestas investigativas.

Apoyando la combinación y procurando aportar en el crecimiento y la difusión de esta aproximación, este documento tiene como objetivo presentar los conceptos principales de las metodologías cuantitativa, cualitativa y mixta, haciendo énfasis en la primera y ejemplificando la determinación de las creencias de los individuos como una base para la construcción de un cuestionario estándar que en un futuro -a través de lo cuantitativo- será utilizado en el estudio de las relaciones entre la motivación y la compartición de conocimiento de las comunidades de práctica de las organizaciones públicas.

Este documento está estructurado en cuatro secciones. La primera caracteriza a la investigación cualitativa y en la segunda se presentan los beneficios y ventajas de lo cuantitativo, cualitativo y lo mixto. Se finaliza con algunas reflexiones, no sin antes ejemplificar el uso de la investigación cualitativa como un complemento y fase previa de lo cuantitativo.

\section{La investigación cualitativa: algunas suposiciones}

La corriente cualitativa tiene sus orígenes en las ciencias sociales, en espacial en la sociología, la antropología cultural de Chicago, el tratado de Berger y Luckmann (1967) y la sociología del conocimiento. En conjunto, estas tendencias representaron un movimiento en contra del positivismo, una reacción a la revolución de los métodos empleados en las ciencias sociales en el siglo XIX y XX (Brower, Abolafia y Carr 2000), que se estimaron inca- 
paces de abordar la complejidad de los fenómenos sociales (López Herrera y Salas Harms 2009).

A la investigación cualitativa se la conoce con una variedad de nombres, incluyendo constructivista (en Brower, Abolafia y Carr 2000). Estas variedades de términos han generado diversos esquemas de clasificación que, además, identifican muchos enfoques de investigación cualitativa. Así, por ejemplo, una sola editorial, Sage, ofrece aproximadamente 140 opciones sobre métodos cualitativos, lo que según el punto de vista podría representar una bendición o un problema.

En suma, los estudios cualitativos van desde la etnografía de casos individuales, mediante comparaciones, hasta grandes conjuntos de información basados en técnicas como recolección de datos, entrevistas, observaciones directas y de participantes, y recopilación y revisión de documentos. Esto da como resultado múltiples formas de datos cualitativos que incluyen citas directas, notas textuales y extractos documentales (Ashworth, McDermott y Currie 2018).

El presente artículo se enfoca en el análisis de contenido de la entrevista, considerada primordialmente como una técnica de interpretación documental -texto, imagen, sonido o cualquier otro registro- que se "basa en la lectura como instrumento de recogida de información, que a diferencia de la lectura común [...] debe ser sistemática, objetiva, replicable y válida" (Abela 2016, 2).

De esta manera, los textos que se analizarán deben entenderse tanto en su manera explícita como en su forma latente; es decir, hay textos ocultos formados de señales e insinuaciones que el entrevistado puede manifestar mientras expresa sus respuestas. A todo esto, el análisis tiene cierta complejidad adicional, ya que el estudio de los datos empieza desde su generación: hay que manejar la relación entre el entrevistador y el entrevistado, en especial respecto a los posibles sesgos.

Por eso se reitera que la aplicación de ese tipo de análisis requiere que las respuestas se entiendan en su sentido literal y más allá, y que es importante considerar la situación del autor del texto y sus circunstancias. Por tanto, una parte metodológica fundamental es obtener la voz de varios colaboradores, de manera que se puedan establecer consensos y controversias que a su vez muestren puntos altos o aristas a considerar en el análisis objeto de estudio. 
Bajo este esquema, incluso una respuesta incompleta o la falta de respuesta puede ser una fuente importante de análisis, más aún cuando se presenta de manera constante durante la recolección de datos.

A nivel general, el análisis de contenido busca estructurar la información que se presenta de forma libre; es decir, el propósito principal de esa lectura profunda es generar elementos de significado que permitan verificar hacia dónde convergen los textos y dónde se alejan. En la mayoría de los casos, esta estructuración se establece a través de la teoría fundamentada, cuya suposición fundamental es que los hallazgos emergen de los datos. Consecuentemente, se procuran identificar unidades de análisis -ideas comunes relacionadas con el objeto de estudio que toman forma de patrón- para después realizar una codificación y categorización, que es el producto final de este tipo de análisis.

La codificación se contrapone con la teoría vinculada al objeto de estudio. Esas ideas-fuerza son definidas completamente, incluyendo su relación con otras similares en la categorización. Estas categorías "permiten asignar significados comunes a la información compilada durante una investigación" (Narváez 2014, 11).

Actualmente existen varias aplicaciones informáticas específicas que facilitan la codificación y categorización del análisis de contenido, no solo mediante la creación de matrices de frecuencia de palabras, sino también mediante la identificación de sinónimos, familias lexicales e incluso esquemas que muestran cómo los predicados se atan a los sujetos definidos por el investigador. Con el uso de estas aplicaciones, la posibilidad de gestionar una gran cantidad de textos de diferente naturaleza se ha elevado exponencialmente, pero no se deben dejar a un lado los criterios de selección y cómo las proposiciones responden a los objetivos de estudio.

En este estudio, se usó el software QSR International, denominado NVivo versión 10, que facilita la organización, el análisis y la búsqueda de perspectivas en datos cualitativos o no estructurados, como la entrevista, las respuestas de preguntas abiertas (encuestas) y los contenidos de la web y redes sociales. Previo a desarrollar un ejemplo, cabe realizar algunas precisiones respecto a los paradigmas de investigación cualitativa y cuantitativa, porque la aplicación práctica propuesta encaja en el marco de las propuestas mixtas, con énfasis en lo cualitativo. 


\section{Lo cualitativo, lo cualitativo y lo mixto}

Parecería que en las ciencias sociales existe una división contundente entre las investigaciones cualitativa y cuantitativa (Ragin, Nagel y White 2004). La investigación cuantitativa asume que la realidad se puede representar objetivamente como abstracción de la vida real, y la investigación cualitativa asume la presencia de realidades múltiples, construidas por varios participantes a medida que participan en sus propias experiencias locales diarias (Brower, Abolafia y Carr 2000).

Otra diferencia radica en que la investigación cualitativa estudia los entornos estructurales-situacionales y la investigación cuantitativa, la asociación entre constructos cuantificados. Evitando la cuantificación, lo cualitativo se diferencia de su par por basarse en descripciones narrativas y hacer uso de técnicas como, por ejemplo, la observación y entrevistas no estructuradas, para -en la medida de lo posible-identificar "la naturaleza profunda de las realidades, su sistema de relaciones, su estructura dinámica" (López Herrera y Salas Harms 2009, 131). Por otro lado, y buscando la generalización de los resultados, lo cuantitativo determina la fuerza de la correlación de las variables a través del estudio de una muestra y la inferencia sobre una población (López Herrera y Salas Harms 2009).

Adicionalmente, y respecto a la forma en que captan el conocimiento, mientras que los investigadores cuantitativos intentan permanecer separados de los participantes para evitar que ellos reaccionen ante su presencia, los investigadores cualitativos creen que la subjetividad es inevitable en las ciencias sociales y consideran que los fenómenos que vale la pena estudiar son aquellos que se consideran significativos precisamente porque los espectadores les otorgan valor.

En otro aspecto, los investigadores cuantitativos introducen la subjetividad incluso en sus elecciones de temas y sitios de estudio, y en las formas en que definen las variables. Ellos persiguen e insisten en que generan datos imparciales y sin valor; en cambio, los investigadores cualitativos resaltan sus orientaciones de valor y las de sus participantes, aunque, al perseguir conclusiones creíbles, intentan mantenerlas en el olvido.

Los informes cuantitativos se suelen escribir en tercera persona, en pasado, presumiblemente para mejorar el sentido de desapego del investigador 
y la representación sin valor; los investigadores cualitativos, en cambio, a menudo usan el tiempo presente y el pronombre plural en primera persona (nosotros) para ayudar a redactar la participación de los lectores en la interpretación de la evidencia que se presenta e iluminar la presencia del investigador en el estudio (Brower, Abolafia y Carr 2000).

A pesar de las diferencias y desde el punto de vista de este estudio, se asume la visión de Ragin, Nagel y White, quienes, más que una dualidad, plantean que las investigaciones cualitativa y cuantitativa se encuentran en "un continuo en el que se pueden identificar dos extremos claramente definidos" $(2004,9)$. En uno de ellos se ubica la primera, que desecha los conceptos de la teoría tradicional aduciendo que es dudosa, y que en el proceso de investigación palidece la distinción en el objeto estudiado y el examinador. En el extremo opuesto, la investigación cuantitativa se caracteriza por considerar la objetividad en el análisis de poblaciones, casos y variables claramente identificadas, asumiendo que las teorías e hipótesis pertinentes están bien especificadas. Pero, además, y entre los dos extremos, existen estrategias de investigación híbridas y combinadas que contemplan tanto métodos cualitativos como cuantitativos.

Los investigadores utilizan a menudo estos métodos mixtos. Por ejemplo, las estrategias cualitativas pueden utilizarse para obtener información sobre el significado, el afecto y la cultura, mientras que las cuantitativas se utilizan para medir características estructurales, contextuales e institucionales. En palabras de Tashakkori y Creswell (2007), en lo mixto el investigador recoge y analiza la información, integra los resultados y realiza inferencias a través de métodos cuantitativos y cualitativos en un mismo estudio. Se trata de combinar la recolección de datos cuantitativos y cualitativos, así como su análisis, en una misma investigación.

Otras combinaciones de enfoques cualitativos y cuantitativos incluyen estrategias híbridas. Este es el caso seleccionado en esta propuesta, pues se usan métodos cualitativos para construir tipologías de narraciones de casos a partir de datos de entrevistas en profundidad, y luego, a futuro, se emplean las narrativas modales como categorías en el análisis cuantitativo.

En general, las investigaciones cualitativas y cuantitativas son enfoques complementarios. Las primeras pueden proporcionar lo que a menudo falta en las segundas, como la evidencia sobre mecanismos y significados. $\mathrm{Mu}-$ 
chas hipótesis pueden eliminarse rápidamente basándose en la investigación cualitativa, al igual que se pueden obtener diversas formas de encontrar tipos específicos de evidencia.

Con la combinación de métodos, la fase cualitativa puede entenderse como un prólogo relativamente económico para una próxima investigación con una gran muestra, es decir, una prueba preliminar informal que refina las hipótesis y las medidas (Ragin, Nagel y White 2004).

Otras combinaciones comunes contemplan el uso de métodos cualitativos en las fases finales de una investigación con una gran muestra, porque los mecanismos causales rara vez son visibles en la investigación cuantitativa convencional, generalmente deben ser inferidos. Así, los métodos cualitativos pueden ser útiles para evaluar la credibilidad de estos mecanismos. Por lo general, estos diseños implican un estudio en profundidad de una submuestra pequeña cuidadosamente seleccionada de los casos del estudio de muestras grandes.

Los casos elegidos se pueden examinar con diferentes grados de detalle, dependiendo de los objetivos del investigador. Los métodos cualitativos empleados en esta etapa van desde la entrevista en profundidad (el complemento cualitativo más común) hasta la observación detallada de la situación y el entorno de cada caso.

Un tercer uso se da cuando es posible integrar técnicas de recopilación de datos cualitativos en un estudio de muestras grandes. Varios investigadores han utilizado dispositivos de narración de historias, a veces de forma casi experimental, para obtener significados de los encuestados y los fenómenos subjetivos relacionados. Si bien estos estudios aún son de naturaleza predominantemente cuantitativa, hay al menos un intento por responder a algunas de las limitaciones de los métodos cuantitativos convencionales.

Finalmente, algunos investigadores intentan el análisis cuantitativo y cualitativo de los mismos casos. Esta estrategia es común cuando las muestras son de tamaño moderado (por ejemplo, una $N$ de 30); se emplean cuando los investigadores buscan demostrar que los resultados de los análisis cuantitativos y cualitativos son complementarios. Con un número moderado de casos, es posible establecer un grado razonable de familiaridad con cada uno, para enfrentar a todos como casos distintos. Al mismo tiempo, la $N$ de casos es suficiente para análisis cuantitativos simples (Ragin, Nagel y White 2004). 
Resumiendo, son muchas las fortalezas y debilidades de lo cuantitativo y lo cualitativo en la investigación social. Existen posturas extremas, pero también hay una visión moderadora que aboga por la complementariedad, es decir, que ambos tipos de investigación son válidos, se apoyan y reconocen mutuamente (McNabb en Blanco-Peck 2006): dada la naturaleza del estudio, se seleccionan y utilizan técnicas de investigación cualitativas y cuantitativas que dan lugar a una visión mixta, lo que permite robustecer las hipótesis y ahondar en los resultados de las investigaciones.

\section{Un ejemplo de lo cualitativo como fase previa a lo cuantitativo}

En el siguiente apartado se expone, mediante un ejemplo, cómo los métodos cualitativos - a manera de prueba preliminar que afine las hipótesis y medidas- pueden ser empleados para resolver un problema concreto de investigación. Para ello, se explican los antecedentes y la teoría empleada, el diseño de la investigación, las entrevistas recopiladas y el método de análisis de datos, con el objetivo de exponer el uso de la investigación empírica en el campo de la administración.

\section{Antecedentes y teoría}

Mediante el uso de la teoría del comportamiento planificado (TPB, por sus siglas del inglés theory of planned behavior) se pretende identificar los posibles factores de fondo que pueden influir en la conducta de compartición de conocimiento de las comunidades de práctica de las organizaciones públicas.

La TPB sostiene que la conducta humana es voluntaria y que los individuos toman decisiones racionales para participar (o no) en una conducta o comportamiento determinado. Las elecciones realizadas están influenciadas por las propias creencias de los individuos sobre el resultado y la evaluación de la ventaja (o desventaja) de involucrarse en el comportamiento objetivo. Estas suposiciones subyacen a tres creencias conceptualmente distintas: con- 
ductuales, normativas y de control. Las primeras se refieren a las creencias percibidas sobre los resultados probables de involucrarse en el comportamiento objetivo y a la evaluación de la conveniencia de estos resultados; las segundas tienen que ver con la presión social percibida; y las últimas tratan sobre la facilidad o dificultad percibida de involucrarse en el comportamiento deseado o no deseado (Ajzen 1991).

Figura 1

Teoría del comportamiento planificado

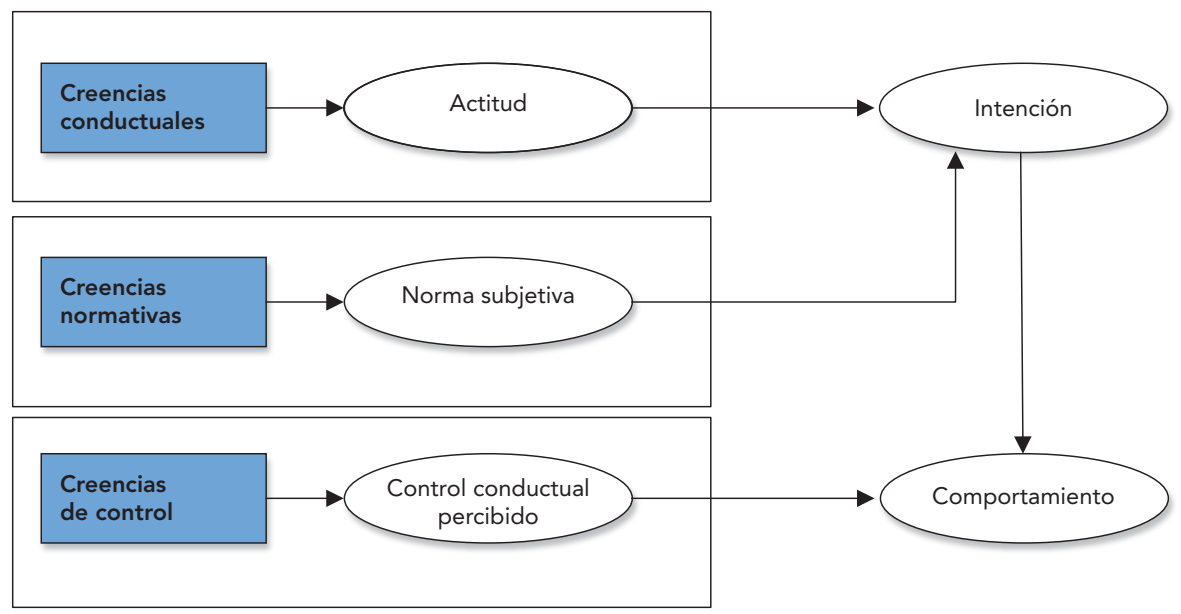

Fuente: adaptado de Ajzen (1991).

En sus respectivos conjuntos, las creencias conductuales generan una actitud hacia el comportamiento, las creencias normativas dan como resultado la norma subjetiva y las creencias de control dan lugar al control conductual percibido. Así, las actitudes reflejan las evaluaciones positivas y negativas de la personas en relación a adoptar una conducta, mientras que las normas subjetivas - o de presión social frente a la realización o no de un acto- muestran la influencia social que siente la gente en relación a las posibles conductas, y el control conductual percibido es la facilidad o dificultad esperada para desarrollar un comportamiento, reflejo de las experiencias 
pasadas de los individuos, así como de una anticipación de impedimentos y obstáculos (Ajzen 1991).

Los pronósticos más cercanos al comportamiento son las intenciones, que a su vez están precedidas por las actitudes, las normas subjetivas y el control conductual percibido. En virtud de ello, cuanto más favorable sea la actitud y la norma subjetiva con respecto a un comportamiento, y cuanto mayor sea el control conductual percibido, más fuerte debe ser la intención de la persona de ejecutar la conducta considerada. La intención es por tanto asumida como el antecedente inmediato de la conducta (Ajzen 1991). Por último, y en la medida en que el control conductual percibido es verídico, puede servir como un poder para el control exacto y contribuir a la predicción de la conducta en cuestión.

En definitiva, la TPB considera que las actitudes hacia un comportamiento determinado, la existencia de normas subjetivas y el control percibido por parte de un individuo son tres antecedentes fundamentales para que la persona defina su intención de comportamiento. La intención -junto con el efecto directo del control conductual percibido- es, entonces, la antesala para el comportamiento.

Para complementar el sustento teórico, se define a las comunidades de práctica (COP, por sus siglas del inglés communities of practice) como grupos de personas, unidas de manera informal por la experiencia compartida y el entusiasmo conjunto por la empresa, que interactúan de forma continua compartiendo su preocupación, sus problemáticas o una pasión por un tema específico, y que profundizan sus conocimientos y experiencias en una determinada área de conocimiento.

En consonancia y en palabras de Wenger $(2001,2)$, "no todo aquello llamado comunidad es una comunidad de práctica [...], son cruciales tres características": un dominio de interés compartido; la comunidad formada alrededor de dicho dominio, sobre el que sus miembros construyen relaciones; y la práctica o el repertorio compartido de recursos (experiencias, historias, herramientas, formas de manejar los problemas, entre otros).

Por su propia naturaleza, las comunidades de práctica albergan actividades y rutinas creativas, relacionadas sobre todo con la reconceptualización de problemas y la toma de decisiones, y añaden valor a las organizaciones (Alborníes 2010). Es por esto que se dice que las COP emergen y sobrevi- 
ven en las más duras condiciones, incluso en las organizaciones más jerárquicas y burocratizadas, como el sector público (Kirkman et al. 2013). En esta línea, Galivene y Kaufman (2006) señalan que el aprendizaje social en el contexto de las comunidades de práctica es un recurso poderoso para la innovación, la formación continua y la gestión del conocimiento en las organizaciones públicas.

Con la creación y divulgación del nuevo conocimiento generado en las COP, se obtiene un efecto de doble beneficio en el corazón de lo público: por un lado, se percibe un crecimiento del compromiso organizacional de los individuos y mayor conocimiento de los procesos y las estrategias institucionales; por el otro, las prácticas de la organización adquieren mayor calidad y se estandarizan (Martínez Marín 2008). Asimismo, las comunidades de práctica favorecen conexiones entre los individuos en las estructuras formales, aunque primero es necesario superar varias cuestiones de organización (Wenger 2001).

Al tiempo que se identifican esas cuestiones, el sector público está reconociendo la importancia de los procesos menos formales de compartir, socializar y crear conocimiento entre pares y dentro de pequeños grupos con intereses afines, reconocimiento que busca aportar en la promoción de cambios y la difusión de la innovación en este tipo de organizaciones. En este contexto, las preguntas clave que se imponen son: ¿cuáles son las creencias que determinan la compartición de conocimiento de las comunidades de práctica de la administración pública?, ¿cuál es la influencia de esas creencias de motivación en los procesos de creación y compartición de conocimiento de las comunidades de práctica de las organizaciones públicas?

El objetivo del presente estudio pretende ofrecer una respuesta a la primera interrogante, a través del uso de la metodología cualitativa. En un futuro y con lo cuantitativo, se solventaría la segunda, considerando que lo cualitativo es una premisa de lo cuantitativo y, por tanto, se recurre y evidencia la utilidad de las metodologías combinadas, híbridas o mixtas. 


\section{Metodología y diseño de la investigación}

Este trabajo utiliza el diseño híbrido, dando prioridad y énfasis a la parte cualitativa. En términos de Morse (1991), cuyo sistema emplea las abreviaturas qual y quan para representar lo cualitativo y cuantitativo, respectivamente, esta investigación es del tipo QUAL, quan. Nótense las mayúsculas para remarcar la predominancia de lo cualitativo.

Por otra parte, se usa el símbolo de la flecha $(\rightarrow)$ por cuanto se trata de un diseño en secuencia QUAL $\rightarrow$ quan: primero lo cualitativo y después lo cuantitativo. Se emplearía el símbolo $(+)$ para indicar un diseño simultáneo, que en este caso no aplica. Según lo mencionado, a esta investigación se la clasifica en el tipo diferente importancia y secuencial QUAL $\rightarrow$ quan.

De acuerdo a Creswell y Plano Clark (en Molina Azorín et al. 2012), este estudio es catalogado como exploratorio porque tiene como propósito, en primer lugar, realizar un análisis previo con el fin de determinar las creencias de motivación, es decir, identificar las medidas y variables más adecuadas que, luego y mediante la construcción de un instrumento para la recogida de datos (encuesta), se utilizarán en la segunda parte de la investigación. El análisis previo se desarrolla mediante una investigación cualitativa, mientras que la segunda parte será la cuantitativa; esto es, estamos ante un diseño secuencial de dos fases.

Con el fin de probar el modelo de investigación propuesto, se adoptó el método de entrevista a profundidad para recopilar los datos y como un procedimiento de provocación de creencias conductuales, normativas y de control, según la TPB. Posteriormente, se examinó la información aplicando el método de la codificación y considerando que la unidad de análisis es el individuo.

Es preciso señalar que la TPB está constituida por variables hipotéticas y predictoras. Las primeras, también denominadas latentes, son los constructos teóricos que conforman la TPB, y no pueden ser observadas directamente, sino inferidas. Las segundas pueden ser valoradas directamente, mediante las respuestas de los individuos y el uso de escalas (Likert, Thurstone, entre otros), como opciones de réplica a las interrogantes planteadas por el evaluador.

A pesar de que se logran descubrimientos interesantes, las mediciones directas de los constructos de la TPB no pueden ser obtenidas mediante el 
uso de preguntas arbitrarias o escogidas en el análisis de estudios previos (aproximación), pues "pueden producirse mediciones con relativamente baja confiabilidad y pueden conducir a una subestimación de las relaciones entre los constructos de la teoría y su validez predictiva" (Ajzen 2002, 3). En ese sentido, para garantizar que las mediciones sean confiables y consistentes, y debido a que hay una infinidad de variables para diferentes comportamientos y poblaciones, es vital seleccionar ítems apropiados en la investigación.

La actitud, la norma subjetiva y el control conductual percibido pueden medirse indirectamente con base en las creencias, pero su identificación requiere de un trabajo previo -haciendo uso de una entrevista- en que se provea a los participantes de una descripción de la conducta de interés y se les pida contestar una serie de preguntas relacionadas con las ventajas y desventajas, los individuos o grupos cuya conducta aprobarían o desaprobarían, y los factores o circunstancias que habilitarían o deshabilitarían llevar a cabo el comportamiento de estudio.

Las respuestas obtenidas son utilizadas para identificar las creencias personales accesibles - es decir, las únicas creencias de cada participante en la investigación- o para construir una lista de las creencias modales accesibles -en otras palabras, las creencias más comúnmente sostenidas en la población investigada (motivo del presente estudio)-. Estas creencias modales y accesibles van a proveer la base para construir el cuestionario estándar, que posteriormente será usado en el estudio principal y que contiene diversos ítems que valorarán un constructo dado (Ajzen 2002).

Es necesario recalcar que el alcance de este documento contempla la identificación de las creencias conductuales de la TPB como ejemplo del uso de estrategias cualitativas y como un trabajo previo para el posterior uso de instrumentos de recolección y técnicas de análisis cuantitativos.

\section{Aplicación y análisis de entrevistas}

Como ya se indicó, mediante la aplicación de entrevistas y el análisis de los datos con la categorización, se identificaron las creencias que eran sobresalientes para la población objetivo (servidores públicos), una vez que fueron 
confrontadas con un escenario específico de compartición de conocimiento en las comunidades de práctica.

La muestra seleccionada está constituida por trece servidores expertos en la materia y que pertenecen al Departamento de Gestión de la Calidad y Cambio Institucional ${ }^{1}$ del Servicio de Rentas Internas (SRI), institución pública del Ecuador con jurisdicción nacional, que tiene como misión gestionar la política tributaria del país. Es decir, es la responsable de la administración y recaudación de los impuestos a nivel nacional.

En todos los casos, las entrevistas fueron realizadas en las oficinas de los expertos, posterior a la planificación telefónica de la cita, en la que se explicó de modo resumido el propósito de la investigación. Su duración media fue de 45 minutos, que corresponde al estándar para entrevistas de este tipo (Coller 2000). Se solicitó a los servidores seleccionados que participaran de forma individual y respondieran a siete preguntas; previamente se les presentó una breve explicación de la conducta de estudio y recibieron unos pocos minutos para organizar sus pensamientos. Todas las entrevistas fueron registradas de forma escrita.

Con ayuda del programa NVivo, se realizó la búsqueda de frecuencias de palabras, con el fin de realizar un primer acercamiento y tener una idea del contenido que más se repite en las entrevistas. Posteriormente, se leyeron y exploraron los textos de cada entrevista para establecer el marco conceptual del estudio mediante el uso de nodos interactivos, es decir, categorizando con base en las creencias del comportamiento. Las respuestas de las dos preguntas iniciales determinaron las creencias conductuales, las de la tercera y cuarta preguntas sirvieron para identificar las creencias normativas, y las de las tres preguntas finales establecieron las creencias de control.

A continuación se presentan las interrogantes y algunas réplicas de los encuestados que sirven de ejemplo.

Para las creencias conductuales:

1. De acuerdo al Estatuto Orgánico de Gestión Organizacional por Procesos del Servicio de Rentas Internas vigente (EC Servicio de Rentas Internas 2016), el Departamento de Gestión de la Calidad y Cambio Institucional es responsable de la base de conocimiento institucional y de las herramientas y proyectos para la gestión del cambio institucional, productos relacionados con las iniciativas de compartición del conocimiento, como es el caso de las comunidades de práctica (Wenger 2001). 
Entrevistador: ¿Cuáles, en su criterio, son las ventajas de compartir conocimiento en las COP del SRI?

Informante A: Tener una red unificada de criterios en la cual cualquier servidor puede acceder a consultar la respuesta emitida a su inquietud, y sobre todo mantener un solo criterio hacia los ciudadanos que demandan atención. Por otro lado, en la discrepancia de criterios, se puede llegar a consensos, basados en la normatividad vigente y en la experiencia de funcionarios con trayectoria institucional. Incluso se puede llegar a levantar, de ser necesario, procedimientos o instructivos que mantengan a los servidores en una línea argumental y una respuesta ciudadana: crecer profesionalmente, lo que suma a que la organización crezca y mejore su imagen interna y externa.

Entrevistador: ¿Cuáles, cree usted, son las desventajas de compartir conocimiento en las COP del SRI?

Informante B: Si la información no es verídica, se puede generar reproceso y repreguntas al conocimiento compartido.

La opinión del informante A puso de manifiesto que las creencias conductuales encajaron en las categorías de las COP como mecanismo que asegura la unificación de criterios y el crecimiento profesional, mientras que con el criterio del informante B se categorizó a las COP como perjudiciales, al considerarlas como espacios de difusión de información errónea no oficial.

La mayor parte de las respuestas relacionadas con las creencias conductuales (40\%) afirmaron que las COP mejoran la ejecución de los procesos en la organización, permiten la unificación de criterios y evitan la fuga del conocimiento en las instituciones. Estos hallazgos no coinciden con la literatura existente.

Autores como Bock et al. $(2005,82)$ y Jeon, Kim y Koh $(2011,267)$ proponen que "lo bueno", "la experiencia agradable", "lo valioso para mí", "un movimiento sabio" y "lo perjudicial" son factores o creencias conductuales que influyen sobre los resultados probables de involucrarse o no en la compartición de conocimiento de las COP.

En relación a las creencias sociales:

Entrevistador: ¿Cuáles individuos o grupos aprobarían que usted comparta conocimiento en las COP?

Informante $\mathrm{C}$ : Considero que tengo el apoyo de mi línea de supervisión directa. 
Entrevistador: ¿Cuáles individuos o grupos desaprobarian que usted no comparta conocimiento en las COP?

Informante D: En mi criterio, las autoridades, mandos altos y medios.

Las respuestas de los informantes $\mathrm{C}$ y $\mathrm{D}$ fueron directas, al igual que la mayoría de los criterios de los entrevistados de la muestra seleccionada (73\%): jefatura inmediata, autoridades y compañeros. Estas categorías coinciden con propuestas de investigación previas, que señalan que los $\mathrm{CEO}$, la jefatura inmediata, los miembros del equipo de trabajo y los líderes son los factores que ejercen presión social a los individuos que participan en una COP (Bock et al. 2005; Jeon, Kim y Koh 2011).

Para terminar, las entrevistas de las creencias de control:

Entrevistador: ¿Cuáles son los factores o circunstancias que le facilitarían compartir conocimiento en las COP del SRI?

Informante E: Creo que el personal altamente capacitado: no hay expertos, o hay falta de habilidad por parte de los expertos para transmitir el conocimiento de manera efectiva. Que los líderes de la organización lo vean como algo estratégico y le den impulso, pues es una ventaja competitiva para la organización.

Entrevistador: ¿Cuáles son los factores o circunstancias que harían difícil o imposible que usted comparta conocimiento en las COP?

Informante F: Falta de disponibilidad presupuestaria para incentivar iniciativas de compartición. Falta de sinergia entre personas. Poca cantidad de capacitadores, facilitadores, expertos.

Entrevistador: ¿Hay otros asuntos que le vengan a la mente cuando piensa en la dificultad de compartir conocimiento en las COP del SRI?

Informante G: Falta de tiempo, no hay prioridad para iniciativas de este tipo. Los expertos no tienen tiempo. No hay un plan de incentivos integral para fomentar el aprendizaje en las personas o participar en procesos de transferencia de conocimientos.

Cuando se analizaron las respuestas de los informantes E, F y G, los criterios vertidos en el primer caso pudieron clasificarse en las categorías carencia de expertos, falta de habilidades de compartición y falta de trabajo en equipo.

En la segunda opinión se evidenciaron las categorías ausencia de recursos financieros y carencia de expertos; y la tercera encajó en las categorías 
excesiva carga laboral y ausencia de una estrategia organizacional para la compartición de conocimientos.

Estos hallazgos se contraponen a propuestas como las de Chau y $\mathrm{Hu}$ (2001) y Jeon, Kim y Koh (2011), que identifican a las habilidades de compartición, provisión de recursos y conocimientos para manejar los sistemas informáticos de soporte como factores que facilitan o dificultan la conducta de compartición de conocimientos.

Parecería que los hallazgos de este estudio no coinciden con la literatura existente, pero, lejos de ser un error, la TPB no especifica las creencias, simplemente apunta a una gran cantidad de posibles factores de fondo que pueden influir en las personas y dependen de los tipos de comportamiento y culturas estudiadas (Ajzen 2011). Se espera, por tanto, que los factores identificados en esta fase cualitativa influyan indirectamente en las intenciones y el comportamiento de compartición de conocimientos de las COP.

De esta manera, se identificaron 25 creencias accesibles conductuales, 7 normativas y 31 de control haciendo uso del software NVivo 10; para ello, se crearon 63 categorías en las que clasificaron las opiniones emitidas por la muestra seleccionada. A continuación, en la tabla 1, se muestran las creencias más importantes con los porcentajes más altos.

\section{Tabla 1}

Creencias conductuales, normativas y de control del SRI

\begin{tabular}{|c|c|c|c|c|}
\hline No. & Nombre & Referencias & Clasificación & Porcentaje \\
\hline 1 & $\begin{array}{l}\text { Mejora en la ejecución } \\
\text { de los procesos en la organización. }\end{array}$ & 12 & \multirow{3}{*}{$\begin{array}{l}\text { Creencias } \\
\text { conductuales }\end{array}$} & $23 \%$ \\
\hline 2 & Unificación de criterios. & 5 & & $9 \%$ \\
\hline 3 & $\begin{array}{l}\text { Evita la fuga del conocimiento } \\
\text { en la organización. }\end{array}$ & 4 & & $8 \%$ \\
\hline 4 & Jefatura inmediata. & 11 & \multirow{4}{*}{$\begin{array}{l}\text { Creencias } \\
\text { normativas }\end{array}$} & $42 \%$ \\
\hline 5 & Autoridades. & 5 & & $19 \%$ \\
\hline 6 & Área de capacitación de la organización. & 3 & & $12 \%$ \\
\hline 7 & Compañeros. & 3 & & $12 \%$ \\
\hline
\end{tabular}




\begin{tabular}{|c|l|c|c|c|}
\hline 8 & $\begin{array}{l}\text { Ausencia de una estrategia } \\
\text { organizacional para la compartición } \\
\text { del conocimiento. }\end{array}$ & 7 & \multirow{2}{*}{$\begin{array}{c}\text { Creencias } \\
\text { de control }\end{array}$} & $13 \%$ \\
\hline 9 & $\begin{array}{l}\text { Excesiva carga laboral, lo que no } \\
\text { permite participar en iniciativas } \\
\text { de compartición del conocimiento. }\end{array}$ & 7 & & $6 \%$ \\
\hline 10 & Carencia de expertos. & 3 & $6 \%$ \\
\hline 11 & $\begin{array}{l}\text { Desconocimiento de los beneficios } \\
\text { de compartir conocimiento. }\end{array}$ & 3 & & 6 \\
\end{tabular}

Fuente y elaboración propia.

Con las creencias personales accesibles seleccionadas, se desarrollará el cuestionario estándar, motivo de otro documento de investigación y que, posterior al procesamiento de los datos y con el uso de métodos cuantitativos, permitirá identificar las relaciones causales entre las actitudes, la intención y la conducta de compartición en las COP.

\section{Reflexiones finales}

La elección del método empleado dependerá de la naturaleza del estudio, del objeto y de sus objetivos. Se podría considerar que, en oposición a lo cuantitativo, en lo cualitativo se presta mayor atención a profundizar y no generalizar los resultados de la investigación. Por ejemplo, los métodos cualitativos se pueden usar para obtener información sobre el significado, el afecto y la cultura, mientras que los métodos cuantitativos se usan para medir características institucionales, de la estructura y del contexto.

La selección de un método mixto posee ventajas metodológicas que enriquecen la investigación. Hay muchas combinaciones posibles y tienen ventajas, tales como ahondar y diversificar la información, otorgar valor metodológico para aceptar o rechazar las teorías empleadas, y brindar la seguridad procedimental para la postulación de la presencia de relaciones causales. Pero como en todo, también existen desventajas; en este caso, en la elección 
del pluralismo metodológico, los peros están relacionados con los costos de la investigación, el tiempo y la duración del trabajo.

Respecto del ejemplo empleado, en este trabajo se describe en forma sucinta el proceso sugerido por Ajzen y su teoría del comportamiento planificado para la identificación de las creencias que posteriormente serán utilizadas en la construcción de un cuestionario que permita evaluar la actitud, la norma subjetiva y la intención de compartir conocimiento en las comunidades de práctica de las organizaciones públicas. Con este trabajo cualitativo y haciendo uso de la categorización, se han identificado las creencias conductuales propias de los ecuatorianos que laboran en el sector público, en contraposición a las malas prácticas de investigadores que asumen que las mediciones directas de los constructos de la TPB (creencias) son adaptaciones de ítems usados en estudios previos.

A pesar de que esta aproximación muchas veces logra hallazgos de interés, puede generar mediciones con baja confiabilidad y conducir a una subestimación de las relaciones entre los constructos de la teoría y su validez. Por lo tanto, se deben usar constructos propios para cada conducta y para cada población predictiva (Ajzen 1991).

Adicionalmente, y dado que en la TPB la información sustantiva más detallada sobre los determinantes de un comportamiento está contenida en las creencias de una persona, esta propuesta no especifica dónde se originaron estas creencias; simplemente apunta a una gran cantidad de posibles factores de fondo que pueden influir en las creencias de los individuos para compartir conocimiento en las COP públicas.

Así, la administración tributaria ecuatoriana, a través de sus expertos, ha identificado principalmente, como mediciones directas apropiadas en la compartición de conocimiento de las COP, a 63 creencias de actitud, normas sociales y control conductual percibido. Esto permitirá que las autoridades y los directivos de las instituciones públicas de nuestro país que quieran impulsar la implementación de COP ejecuten acciones diferentes y estrategias que consideren dichas creencias. 
Una aproximación a lo cualitativo: identificando las creencias de la compartición de conocimiento

\section{Referencias}

Abela, Jaime. 2016. "Las técnicas de análisis de contenido: una revisión actualizada". Marcelo Astorga. Accedido 2 de mayo de 2019. https://bit.ly/2D4dk0e

Ajzen, Icek. 1991. "The Theory of Planned Behavior". Organizational Behavior and Human Decision Processes 50 (2): 179-211. https://doi.org/10.1016/0749-5978(91)90020-T

---.2002. "Constructing a TpB Questionnaire: Conceptual and Methodological Considerations". University of Massachussets Amherst. Accedido 20 enero de 2018. https://bit. ly/2PWsHOg

---. 2011. "Behavioral Interventions Based on the Theory of Planned Behavior". University of Massachussets Amherst. Accedido 15 septiembre de 2015. https://bit.ly/2rX5dAL

Alborníes, Ángel L. 2010. La disciplina de la innovación: rutinas creativas. Madrid: Díaz de Santos.

Ashworth, Rachel Elizabeth, Aoife Mary McDermott y Graeme Currie. 2018. "Theorizing from Qualitative Research in Public Administration: Plurality through a Combination of Rigor and Richness". Journal of Public Administration Research and Theory 29 (2): 1-16. https://doi.org/10.1093/jopart/muy057

Berger, Peter, y Thomas Luckmann. 1967. “Aspects Sociologiques Du Pluralisme”. Archives de Sociologie des Religions 12 (23): 117-127. www.jstor.org/stable/30117775

Blanco-Peck, Richard. 2006. "Los enfoques metodológicos y la administración pública moderna”. Cinta de Moebio 27 (8): 256-265. https://bit.ly/2D9e8Ru

Bock, Gee-Woo, Robert W. Zmud, Young-Gul Kim y Jae-Nam Lee Lee. 2005. "Behavioral Intention Formation in Knowledge Sharing”. MIS Quarterly 29 (1): 87-111. https://doi. org/10.2307/25148669

Brower, Ralph S., Mitchel Y. Abolafia y Jared B. Carr. 2000. “On Improving Qualitative Methods in Public Administration Research”. Administration \& Society 32 (42): 363-397. https://doi.org/10.1177/00953990022019470

Cassell, Catherine, y Gillian Symon. 2006. "Taking Qualitative Methods in Organization and Management Research Seriously". Qualitative Research in Organizations and Management: An International Journal 1 (1): 4-12. https://doi.org/10.1108/17465640610666606

---. 2015. "Qualitative Research in Organizations and Management: Ten Years on”. Qualitative Research in Organizations and Management: An International Journal 10 (4): 1-11. https://doi.org/10.1108/QROM-10-2015-1329

Chau, Patrick Y. K., y Jen-Hwa Paul Hu. 2001. "Information Technology Acceptance by Individual Professionals: A Model Comparison Approach”. Decision Sciences 32 (4): 699719. https://doi.org/10.1111/j.1540-5915.2001.tb00978.x

Coller, Xavier. 2000. Cuadernos metodológicos: estudios de casos. Madrid: CIS.

Dodge, Jennifer, Sonia M. Ospina y Erica Gabrielle Foldy. 2005. "Integrating Rigor and Relevance in Public Administration Scholarship: The Contribution of Narrative Enquiry". Public Administration Review 65 (3): 286-300. https://doi.org/10.1111/j.1540-6210.2005.0 0454.x 
EC Servicio de Rentas Internas. 2016. Plan estratégico organizacional 2016-2019. Quito: SRI. http://bit.ly/33fveaV

Galivene, Graciela, y Ester Kaufman. 2006. "Training and Articulating Public Agencies in Argentina". En Encyclopedia of Communities of Practice in Information and Knowledge Management, editado por Elayne Coakes y Steve Clarke, 537-543. Hershey, Idea Group Reference.

Haverland, Markus, y Dvora Yanow. 2012. "A Hitchhiker's Guide to the Public Administration Research Universe: Surviving Conversations on Methodologies and Methods". Public Administration Review 72 (3): 401-408. https://doi.org/10.1111/j.1540-6210.2011.02524.x

Jeon, Su-Hwan, Young-Gul Kim y Joon Koh. 2011. "Individual, Social, and Organizational Contexts for Active Knowledge Sharing in Communities of Practice". Expert Systems with Applications 38 (10): 12423-12431. https://doi.org/10.1016/j.eswa.2011.04.023

Kirkman, Bradley L., John L. Cordery, John Mathieu, Benson Rosen y Michael Kukenberger. 2013. "Global Organizational Communities of Practice: The Effects of Nationality Diversity, Psychological Safety, and Media Richness on Community Performance". Human Relations 66 (3): 333-362. https://doi.org/10.1177/0018726712464076

López Herrera, Francisco, y Héctor Salas Harms. 2009. "La investigación cualitativa en administración”. Cinta de Moebio 35: 128-145. https://doi.org/10.4067/s0717-554x2009000200004

Martínez Marín, Jesús. 2008. "Guía para la correcta implantación de comunidades de práctica en entornos de administración pública: una experiencia de éxito". Red Peruana de Gestores de la Educación. Accedido 18 de agosto de 2012. https://bit.ly/37ol9Ml

Molina Azorín, José Francisco, María Dolores López Gamero, Jorge Pereira Moliner, Eva María Pertusa Ortega y Juan José Tarí Guilló. 2012. "Métodos híbridos de investigación y dirección de empresas: ventajas e implicaciones". Cuadernos de Economía y Dirección de la Empresa 15 (2): 55-62. https://doi.org/10.1016/j.cede.2012.01.001

Morse, Janice M. 1991. "Approaches to Qualitative-Quantitative Methodological Triangulation”. Nursing Research 40 (2): 120-123. https://bit.ly/2s1OBay

Narváez, Guillermo. 2014. Análisis cualitativo, unidades de análisis: presentación de curso. Barcelona: Universidad de Barcelona.

Ospina, Sonia M., Marc Esteve y Seulki Lee. 2017. "Assessing Qualitative Studies in Public Administration Research". Public Administration Review 78 (4): 593-605. https://doi. org $/ 10.1111 /$ puar. 12837

Ragin, Charles C., Joan Nagel y Patricia White. 2004. Workshop on Scientific Foundations of Qualitative Research. Arlington: National Science Foundation. https://bit.ly/37rh7CO

Tashakkori, Abbas, y John W. Creswell. 2007. "The New Era of Mixed Methods". Journal of Mixed Methods Research 1 (1): 3-7. https://doi.org/10.1177/2345678906293042

Wenger, Etienne. 2001. Comunidades de práctica: aprendizaje, significado e identidad. Buenos Aires: Paidós Ibérica. 